\title{
Development of a beam of very slow polarized muons
}

\author{
E. Morenzoni ${ }^{a}$, M. Birke ${ }^{\text {a,d }}$, A. Hofer ${ }^{\text {a,c }}$, F. Kottmann ${ }^{e}$, \\ J. Litterst ${ }^{d}$, B. Matthias ${ }^{b}$, M. Meyberg ${ }^{a}$, Ch. Niedermayer ${ }^{c}$, Th. Prokscha ${ }^{b, a}$, \\ G. Schatz ${ }^{\mathrm{c}}$ and Th. Wutzke ${ }^{\mathrm{a}}$ \\ ${ }^{a}$ Paul Scherrer Institut, $\mathrm{CH}-5232$ Villigen PSI, Switzerland \\ b Physik Institut, Universität Heidelberg, Philosophenweg 12, D-69120 Heidelberg, Germany \\ ${ }^{c}$ Fakultät für Physik, Universität Konstanz, D-78434 Konstanz, Germany \\ 'Institut für Metallphysik und Nukleare Festkörperphysik, Technische Universität Braunschweig, \\ D-38106 Braunschweig, Germany \\ 'Institut für Teilchenphysik, ETH Zürich, CH-5232 Villigen PSI, Switzerland
}

\begin{abstract}
During the last few decades, a variety of methods has been developed which makes use of polarized positive muons as a microscopic probe of the magnetic properties of condensed matter (muon spin rotation, relaxation, resonance, $\mu \mathrm{SR}$ ). Until now, available beams for $\mu \mathrm{SR}$ studies have delivered $100 \%$ polarized muons with energies in the MeV range, resulting in a deep penetration of the muons into the sample material under investigation. This presently limits the applications of the $\mu \mathrm{SR}$ technique to the study of the bulk characteristics of matter. To be able to control the implantation depth, a very low energy beam of polarized muons is being developed at the Paul Scherrer Institute. Very slow polarized muons (kinetic energy $\sim 10 \mathrm{eV}$, polarization $\sim 90 \%$ ) are obtained from the moderation of a high energy muon beam in a thin film of an appropriate condensed gas. These muons can be used as a source for a beam of tunable energy between a few tens of $\mathrm{eV}$ and some tens of $\mathrm{keV}$. Implantation depths in the range of few to a few hundreds of nanometers can thus be achieved by varying the energy.
\end{abstract}

\section{Introduction}

A beam of polarized positive muons $\left(\mu^{+}\right)$with a tunable energy between $10 \mathrm{eV}$ and a few tens of $\mathrm{keV}$ would provide a new powerful instrument for fundamental and applied muon physics studies. Particularly wide and interesting are the new possibilities offered by such a beam to the investigation of condensed matter by muon spin rotation techniques ( $\mu \mathrm{SR}$ ) [1]. This technique, with several variants, has been developed into a powerful research tool during the last decade and some recent results are reported in this conference. It makes use of polarized positive muons and muonium atoms implanted into a sample. There they act as microscopic magnetic probes and allow the study of strength, direction and variations of the local magnetic fields at the stopping sites of the muon or muonium $\left(\mu^{+} e^{--}\right)$. These particles are also used to study diffusion processes, to obtain information about defects and as probes in free radical chemistry. Muon spin research is presently limited to 
the study of bulk characteristics of matter since the range and range straggling of the routinely used, so-called surface $\mu^{+}$(kinetic energy $E \approx 4 \mathrm{MeV}$ ) are approximately $150 \mathrm{mg} / \mathrm{cm}^{2}$ and several tens of $\mathrm{mg} / \mathrm{cm}^{2}$, respectively. To achieve implantation depths between typically fractions of a nm and a few hundred nm, polarized muons with variable energy between $\sim 10 \mathrm{eV}$ and a few tens of $\mathrm{keV}$ are needed. With the availability of such particles thin films, solid-solid and vacuum-solid interfaces would become accessible to $\mu$ SR studies thus offering a new investigative tool to the growing field of thin film and surface physics. The relevance of very slow polarized muons has been recognized for several years and various methods to obtain them have been proposed [2-6]. A method, which is technologically relatively simple, is the moderation technique where energetic particles produced by some source are slowed down in an appropriate material.

At the Paul Scherrer Institute (PSI) we are investigating the technique of decelerating a fraction of an existing intense secondary beam of surface $\mu^{+}$from an initial energy of $\sim 4 \mathrm{MeV}$ to $\sim 10 \mathrm{eV}$ using appropriate insulators. The moderated particles can then be used as a source of a beam of low energy $\mu^{+}$with tunable kinetic energy in the desired range. In this paper we describe the experimental setup, summarize the measured characteristics of the moderated muons and present the status of the development together with some future plans.

\section{Experimental}

The apparatus used for the ongoing studies and developments is shown in fig. 1 . It allows the investigation of the characteristics and physics of the muon moderation process and the use of very slow $\mu^{+}$for first applications. It also serves as a prototype for a future facility to be permanently installed at a secondary beamline of the PSI accelerator to provide a tertiary beam of very slow muons for a large variety of experiments. The central component is an ultrahigh vacuum (UHV) chamber designed for a base pressure of $\sim 10^{-10} \mathrm{mbar}$. As in the case of positrons, the most promising moderator for muons appears to be a condensed van der Waals gas [7,8]. The moderator consists of an Al substrate (250 $\mu \mathrm{m}$ thick, $99.999 \%$ pure), which can be cooled by a helium cryostat to $\sim 6 \mathrm{~K}$, and where a solid layer of the gas, a few $100 \mathrm{~nm}$ thick, is deposited under controlled conditions. The UHV environment is needed to avoid degradation of the moderation efficiency which arises from the deposition of one or more layers of rest gas molecules on the surface. The UHV conditions needed by the moderator are also compatible with the vacuum requirement of surface and thin film studies. In the experiment, a secondary beam line of the PSI accelerator complex is tuned to deliver surface muons. After collimation, the incident surface $\mu^{+}$traverse a thin $(200 \mu \mathrm{m})$ scintillator (S1). They pass through a $50 \mu \mathrm{m}$ stainless-steel window, which separates the UHV of the slow $\mu^{+}$apparatus from the poorer vacuum $\left(\sim 10^{-5} \mathrm{mbar}\right)$ of the beam line and enter the UHV chamber. 


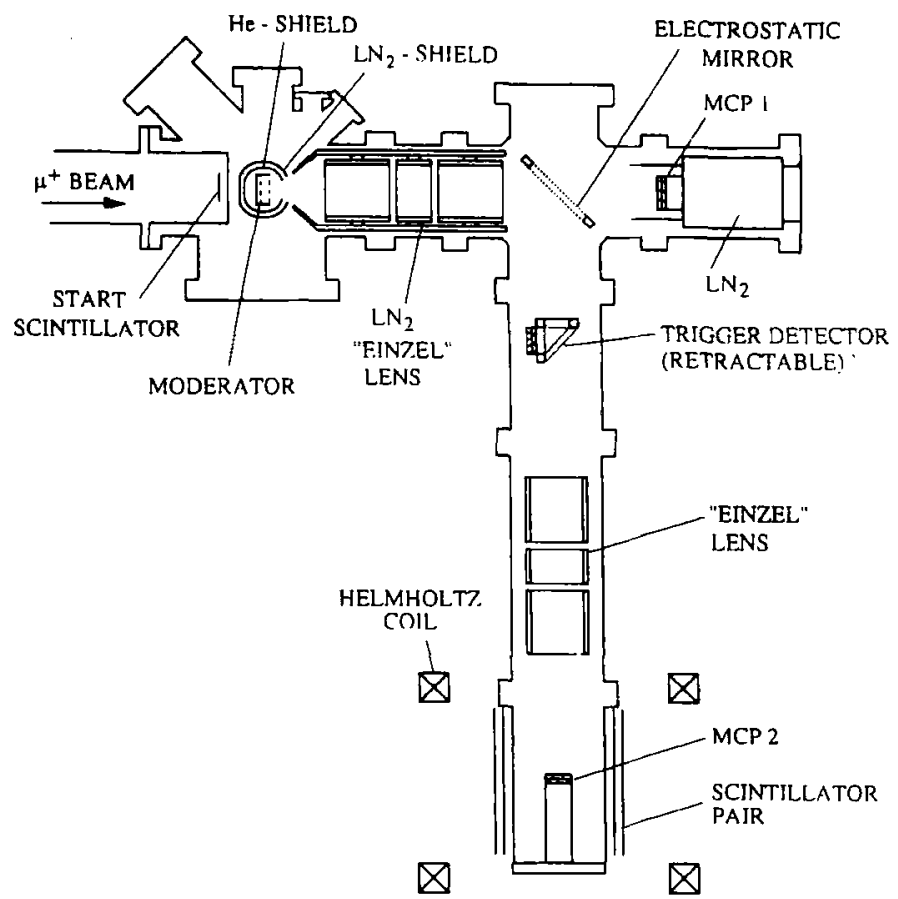

Fig. 1. UHV apparatus with cryostat used for the moderation studies and the very slow $\mu^{+}$ production.

In the moderator, about $50 \%$ of the beam is stopped and a small but not negligible fraction of the $\mu^{+}$are emitted from its downstream side with an energy of several $\mathrm{eV}$. The cold metal substrate is electrically insulated and can be held at a voltage as high as $20 \mathrm{kV}$ to accelerate and extract the very slow $\mu^{+}$from the production region. The acceleration takes place in a two-stage system formed by the substrate and two accelerating grids. The slow muons are deflected by an electrostatic mirror $90^{\circ}$ with respect to the incoming beam and are focussed by two electrostatic "einzel lenses" at a position where a microchannel plate detector (MCP 2) is located. Alternatively, a sample holder can be mounted in place of the MCP. Muons which are not completely moderated are not deflected appreciably by the electrostatic mirror and are detected by another microchannel plate detector (MCP 1), which is used for monitoring and beam tuning purposes. Between the electrostatic mirror and the second "einzel lens" a very thin detector giving a signal when a few keV $\mu^{+}$traverses it can be inserted. This detector (called trigger detector) is essential for $\mu \mathrm{SR}$ and other applications and is explained in more detail in section 4.

The $\mathrm{e}^{+}$emitted by the decay of the $\mu^{+}$stopped at MCP 2 are observed by four pairs of plastic scintillator surrounding MCP 2. With a pair of Helmholtz coils, a magnetic field of up to $200 \mathrm{G}$ can be applied parallel to the direction of the slow muons. 


\section{Results and discussion}

We briefly summarize here the most important results. Details can be found in refs. $[9,10]$. Questions which have been addressed up to now can be summarized as follows:

- Which moderator gives highest yields of very slow $\mu^{+}$?

- Which parameters influence the moderation?

- What are the physical processes governing the emission of very slow $\mu^{+}$?

- What are the characteristics of the moderated muons?

- Are they polarized?

Yields for different moderators as a function of various parameters and energy spectra of the slow $\mu^{+}$were measured with a time-of-flight (TOF) technique. For this measurement the trigger detector was not used and was retracted from the beam axis. The start signal of the TOF measurement is produced by the incoming surface muons as they traverse the thin scintillator $\mathrm{S} 1$. The stop signal is given by the slow $\mu^{+}$accelerated and stopped in MCP 2 (S1-MCP 2 TOF). The muons can be unambiguously identified using the signal of MCP 2 in coincidence with the delayed signal from one of the four pairs of scintillator paddles. Fig. 2 shows the TOF spectrum between S1 and MCP 2. For the measurement of the energy spec-

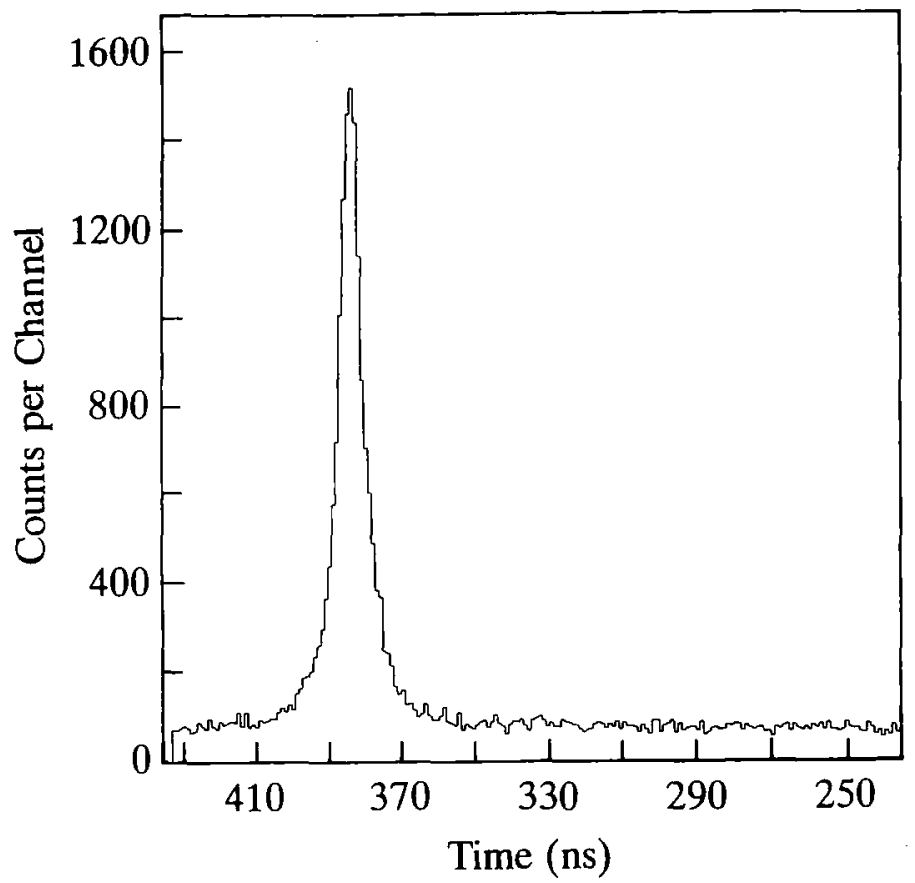

Fig. 2. Time-of-flight distribution between start scintillator and MCP 2 . The peak is given by the very slow $\mu^{+}$emitted from the Ar moderator. 
trum of the moderated muons, a small voltage difference between the substrate and the first grid is applied (electric field $\sim 10 \mathrm{~V} / \mathrm{cm}$ ), so that the muons were only smoothly accelerated and the TOF distribution was broadened. This enhances the sensitivity of the measurement. The transport properties of the electrostatic elements have been studied by means of a Monte Carlo program calculating trajectories and time-of-flights of muons from the moderator surface to MCP 2 for different values of the $\mu^{+}$emission energy. The energy spectrum is obtained by comparing the experimental data with results from the Monte Carlo simulation. The energy distribution of these muons shows a maximum at $\sim 6 \mathrm{eV}$ with a FWHM of $\sim 15 \mathrm{eV}$ and a tail extending to higher energies up to $\sim 100 \mathrm{eV}$. The area of the peak in the TOF spectrum, normalized to the number of incoming surface muons and corrected for transport and detection efficiencies, gives the moderation efficiency of an energetic muon beam with defined momentum and momentum bite. We have investigated various condensed van der Waals gases: rare gases such as $\mathrm{Ne}, \mathrm{Ar}, \mathrm{Kr}$ and $\mathrm{Xe}$ and molecular gases such as oxygen, nitrogen and methane. So far, the most efficient moderators appear to be solid $\mathrm{Ne}$, Ar or $\mathrm{N}_{2}$ deposited on a metal substrate, giving an efficiency, under optimized conditions, of up to $1.5 \times 10^{-4}$ for $\mathrm{Ne}$ for converting an incoming surface $\mu^{+}(p \approx 27.3 \mathrm{MeV} / c$, $\Delta p / p \approx 1.5 \%$ ) into a very slow one. The efficiency for Ar and $\mathrm{N}_{2}$ are a factor 2 to 3 smaller than for $\mathrm{Ne}$.

To optimize the efficiency the influence of parameters such as substrate temperature and growth rate of the film of condensed gas have also been investigated. By measuring the moderation efficiency as a function of the layer thickness, the escape depth of the very slow muons in Ar and $\mathrm{Kr}$ is found to be $\sim 90$ and $\sim 60 \mathrm{~nm}$ respectively. At a pressure of $10^{-10}$ mbar the yield of the slow muons is constant over a period of several days. This demonstrates that the contamination of the moderator, by the sticking of rest gas molecules to its surface, and the effects of muon irradiation of the moderator do not play a major role under these conditions.

A very important property of the slow muons is their polarization. Although in principle information on magnetic moments of the surface can also be obtained with unpolarized particles [11] (for instance by studying the scattering of slow muons at grazing incidence [6]), it is evident that, for an unrestricted use of moderated muons as magnetic microprobes in thin-film and surface studies by the $\mu \mathrm{SR}$ method, they should be polarized.

The polarization of the very slow $\mu^{+}$was measured by using MCP 2 as an active target where the muons stop, give a signal and precess under the influence of a transverse magnetic field [12]. This measurement was also performed without trigger detector. Note that the electrostatic fields of the transport system, at these energies, do not alter the spin direction of the muons in the laboratory frame. So one expects any remaining polarization to be antiparallel to the incident beam direction, i.e. parallel to the surface of MCP 2. To acquire the characteristic $\mu^{+}$spin precession signal, we measure the time between a pulse in MCP 2 and a signal in one of the four $\mathrm{e}^{+}$scintillator pairs. 
For the polarization measurement we select from the S1-MCP 2 TOF spectrum only those events contained in the peak and we correct for the contribution from the flat background produced by random coincidences between S1 and MCP 2. A resulting $\mu$ SR spectrum obtained from $\mu^{+}$moderated in a $\mathrm{N}_{2}$ film and showing the $\mu^{+}$spin precession signal in a transverse magnetic field of $50 \mathrm{G}$ is plotted in fig. 3 . The amplitude of the precession signal is a direct measure of the polarization of the very slow muons. By taking into account the behavior of a polarized $\mu^{+}$stopped in the microchannel plate detector and the sensitivity of our $\mu \mathrm{SR}$ spectrometer we obtain the following values for the absolute polarization of the very slow $\mu^{+}$: for $\mathrm{Ar}$ $(87 \pm 3 \%)$, for $\mathrm{Kr}(80 \pm 20 \%)$ and for $\mathrm{N}_{2}(76 \pm 14 \%)$.

The results show that the very slow $\mu^{+}$obtained by the moderation technique nearly retain their initial $100 \%$ polarization. The high polarization, the energy distribution, the large escape depth and high yields for emission from van der Waals insulators all strongly suggest that the observed very slow $\mu^{+}$are epithermal, i.e. $\mu^{+}$emitted from the moderator before thermalization (hot muon emission). Depending on the energy, different processes are responsible for the slowing down of the $\mu^{+}$in the moderator. Initially the surface $\mu^{+}$rapidly lose energy by Coulomb collisions with electrons, ionizing and exciting the target atoms. When a $\mu^{+}$has lost most of its energy, at energies below $\sim 10 \mathrm{keV}$, charge changing cycles invol-

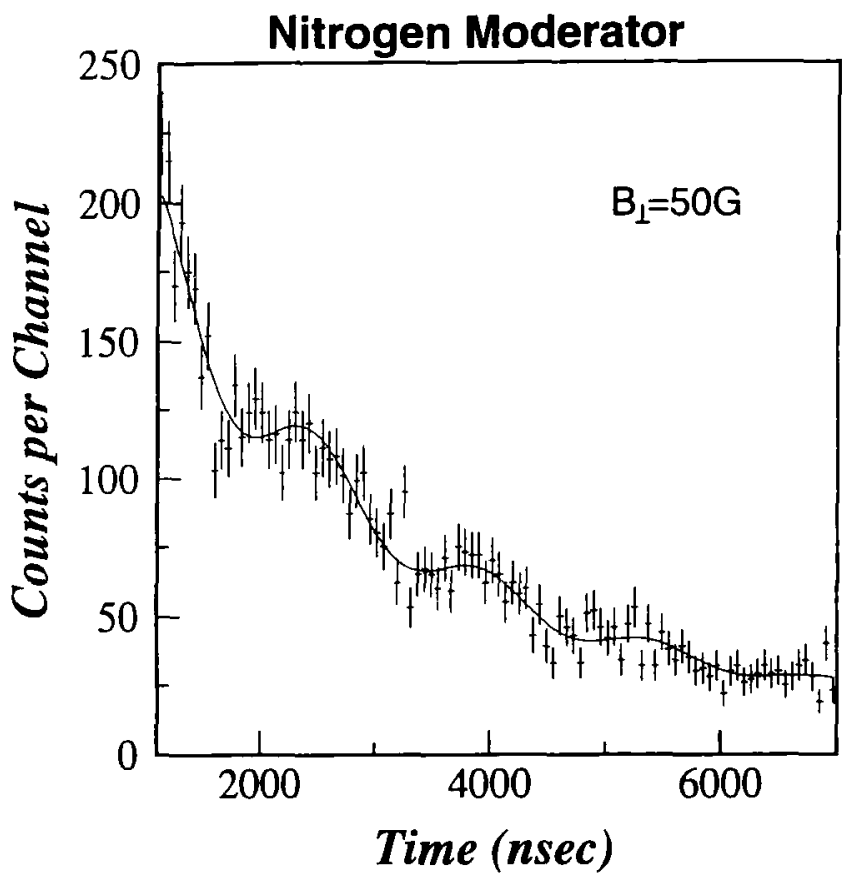

Fig. 3. Decay time distribution of the very slow $\mu^{+}$emitted from a solid $\mathrm{N}_{2}$ layer and precessing in a $50 \mathrm{G}$ transverse magnetic field. 
ving muonium formation and break-up also become important as an energy dissipating mechanism [13]. In wide band gap insulators such as solid $\mathrm{Ne}, \mathrm{Ar}$ and $\mathrm{N}_{2}$, these processes have positive threshold energies (band gap energy 21, 14 and $14 \mathrm{eV}$ [14]). Once the $\mu^{+}$has reached a kinetic energy of the order of these threshold levels, the corresponding efficient energy loss mechanisms are strongly suppressed or even energetically impossible and the energy loss rate becomes low since the the only remaining energy loss mechanism is via relatively inefficient elastic collisions or phonon emission. Energy loss by elastic collisions may be up to several orders of magnitudes less than by electronic excitation processes. Regarding phonon emission, van der Waals solids such as rare gas solids and $\alpha-\mathrm{N}_{2}$ are also particularly favorable to a low energy loss rate since they have soft phonon spectra with energies of the order of 5-10 meV [14] and they do not sustain optical phonons. The particularly efficient moderation to $\mathrm{eV}$ energies of $\mu^{+}$insulators is therefore a consequence of an open energy window in the moderation process which significantly increases the escape depth of the muons once they have reached this energy region. Moreover, the observation that the very slow $\mu^{+}$retain almost the full initial polarization sets an upper limit to the time scale of the mechanism responsible for the moderation and is also consistent with the hot muon emission mechanism. Diffusion-like processes, which assume thermalization at the onset, or delayed processes, where the $\mu^{+}$originates from a muonium atom living for a time scale characteristic of the hyperfine frequency ( $224 \mathrm{ps}$ ), can be excluded as a source of the majority of those particles. In solid Ar and $\mathrm{Kr}$, thermalization leads to practically $100 \%$ [15] and in solid Ne to more than $80 \%$ muonium formation [16]. Muonium survival for times non-negligible compared to the period of the electron and muon spin exchange oscillation due to the hyperfine interaction would reduce the polarization of the very slow $\mu^{+}$by a factor of two, at variance with our results. In solid $\mathrm{N}_{2}$ also, thermalized $\mu^{+}$quickly depolarize and only retain one third of the initial polarization [17]. On the other hand, epithermal $\mu^{+}$emission conserves the initial surface $\mu^{+}$ polarization since depolarization via electron scattering and Coulomb scattering is negligible and the overall time for slowing down to $\sim 10 \mathrm{eV}$ is very short $(\sim 10 \mathrm{ps})$. As a result even muonium formation does not give rise to a sizable depolarization.

\section{Trigger detector for very slow muons}

Various experiments using very slow muons need a clean trigger signal. In particular, for a $\mu$ SR time differential measurement, a signal starting the measurement of the temporal variation of the muon spin must be provided; additionally, unique correspondence between an incident muon and a decay $\mathrm{e}^{+}$must be ensured. In a pulsed machine producing a bunch of particles with narrow time structure, such a signal can be obtained from the high frequency system of the accelerator or by using the signal produced by the muon bunch traversing a scintillator placed upstream 
of the moderator. With a continuous beam such as the one delivered by the PSI cyclotron, the entrance scintillator cannot be used to provide an efficient start signal for a $\mu$ SR measurement, unless an unacceptable reduction of the intensity of the incoming muon beam is taken into account. To obtain a clean signal we have developed a detector (trigger detector) that registers the moderated muons accelerated to typically $10-20 \mathrm{keV}$. Good time resolution, good efficiency and minimal influence on the trajectory of the muons by the detection process are required. The detector is shown in fig. 4. It is placed between the electrostatic mirror and the second electrostatic lens (see fig. 1). It consists of a very thin carbon foil (thickness $\sim 2.7 \mu \mathrm{g} / \mathrm{cm}^{2}$ ) combined with a MCP located perpendicularly to the foil. The slow $\mu^{+}$traversing the thin foil eject one or more secondary electrons with large probability [18]. These electrons are directed by a small grid system to the microchannel plate where they are detected. The amount of material interacting with the muons is, besides the grids, only given by the $\sim 60$ atomic layers forming the foil. Recent measurements indicate that the detector fulfils the requirements. Fig. 5 shows for instance a TOF spectrum obtained with this detector. Muons with $15 \mathrm{keV}$ kinetic energy traverse the foil producing the start signal in the trigger detector and stop in MCP 2 thus providing the stop signal. Two peaks are visible. The more intense one corresponds to slow muons. The small peak on the left side of the $\mu^{+}$peak is produced by those slow muons which capture an electron in the foil and continue as muonium. Note that this spectrum has been obtained with the "einzel lens" switched off. Therefore it directly reproduces the fraction of muonium produced at the trigger foil. With the lens focussing the very slow $\mu^{+}$on MCP 2, their fraction

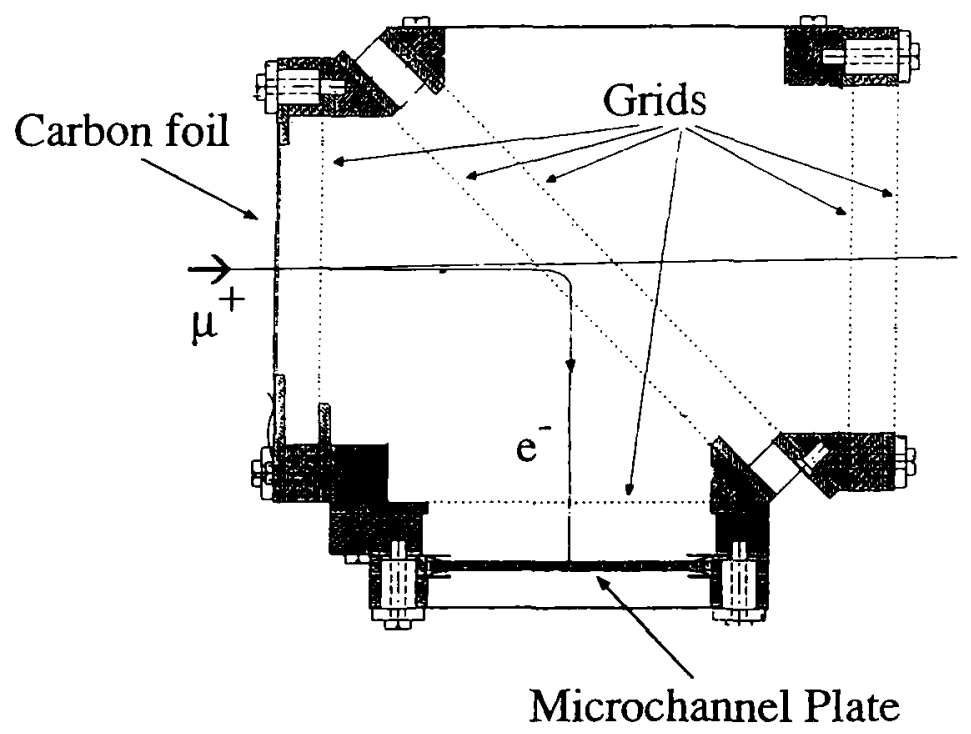

Fig. 4. Trigger detector for keV muons. 


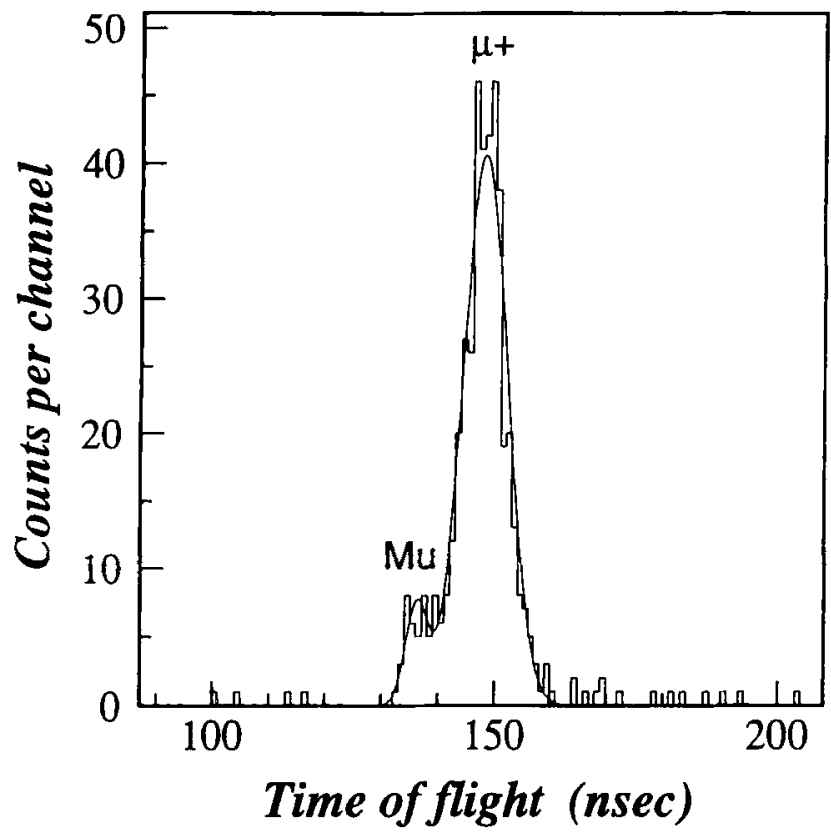

Fig. 5. Time-of-flight between trigger detector and MCP 2 of muons with $15 \mathrm{keV}$ energy.

is increased by a factor $\sim 4$. Planned improvements which are outlined in the next section will reduce the number of muonium atoms reaching the sample to a negligible factor.

Very recently we have performed a first $\mu$ SR measurement of a thin sample with very slow muons. Fig. 6 shows the $\mu$ SR spectrum obtained by implanting $9 \mathrm{keV}$ muons in a $100 \mathrm{~nm}$ thick $\mathrm{SiO}_{2}$ layer deposited on an aluminum substrate. The sample was placed at the MCP 2 position. A transverse magnetic field of $50 \mathrm{G}$ was applied. The start signal was obtained from the trigger detector and the stop signal from one of the $\mathrm{e}^{+}$scintillator pairs. This result demonstrates that first investigations of thin-film samples can be performed with the present setup and gives also an example of the operation mode required by routine $\mu \mathrm{SR}$ measurement in a future $\mu \mathrm{SR}$ facility.

\section{Future plans}

To exploit fully the possibilities offered by the moderation technique in conjunction with an intense secondary beam and to build a tertiary beam further developments are necessary. One of the most important is the development of a section allowing the $\mu^{+}$energy to be tuned in the range between $\sim 6 \mathrm{eV}$ and $30 \mathrm{keV}$. In the present experimental setup the lowest attainable energy of the muons before they 


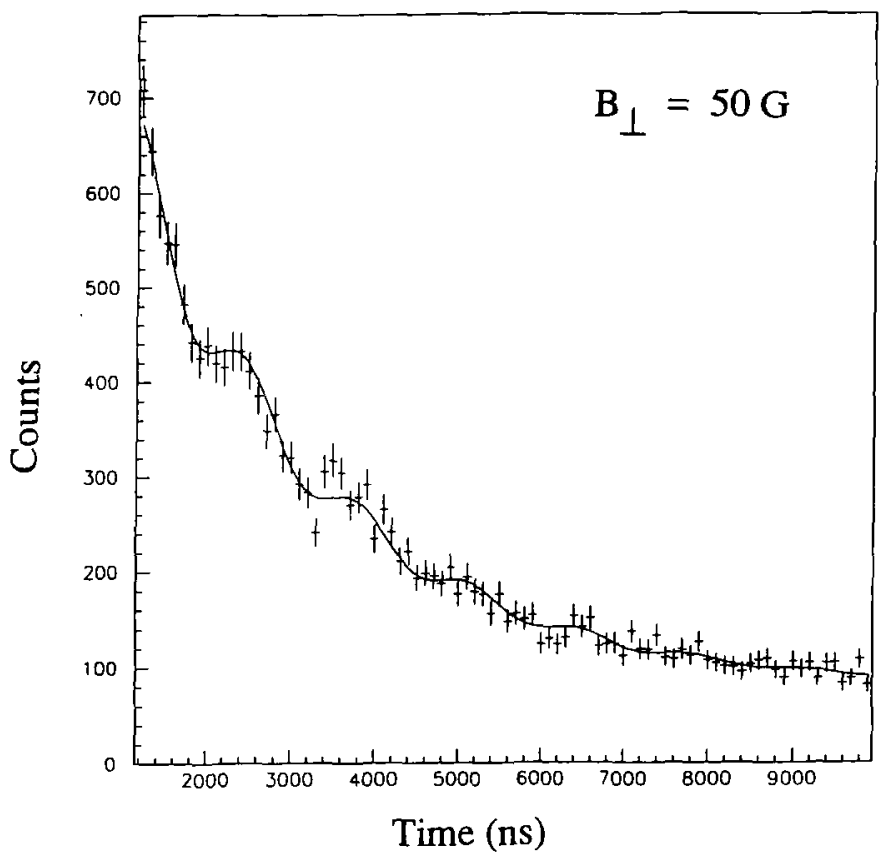

Fig. 6. Decay time distribution of $9 \mathrm{keV}$ muons stopped in a $100 \mathrm{~nm} \mathrm{SiO}$ layer on an $\mathrm{Al}$ substrate and precessing in a $50 \mathrm{G}$ transverse magnetic field.

reach the sample is limited by the presence of the trigger detector. The finite foil thickness sets a lower limit of $\sim 8 \mathrm{keV}$. Below this value, energy loss, energy loss straggling, angular straggling and muonium formation in the foil considerably affect the quality and the intensity of the slow $\mu^{+}$beam. Even without a trigger detector (as is possible at a pulsed accelerator) the $\mu^{+}$lifetime and the finite distance between sample and moderator sets another lower limit of $\sim 1 \mathrm{keV}$. The upper energy (presently $20 \mathrm{keV}$ ) is determined by the requirement of reliable operation of the cryostat with moderator held at this high voltage. To extend the available energy range the insertion of an acceleration-deceleration section between moderator and sample and a new cryostat design are planned. The part of the UHV chamber between moderator and sample which includes the two "einzel lenses", mirror and trigger detector will be electrically insulated and set to a negative high voltage of up to $30 \mathrm{kV}$. In this way, the moderated muons emitted from the condensed film can immediately be accelerated to $30 \mathrm{keV}$ independent of their initial energy. In front of the sample they return to their initial kinetic energy which is chosen by varying the value of the high voltage of the moderator. A new improved cryostat with larger acceptance of the incoming secondary muon beam will allow energies up to $30 \mathrm{keV}$. This scheme requires a major rebuild of the apparatus but allows the tuning of the $\mu^{+}$energy between $\sim 6 \mathrm{eV}$ and $30 \mathrm{keV}$ without restrictions. The fact 
that all the muons traverse the acceleration-deceleration section with the same energy is also advantageous, since the parameters of the transport system and of the trigger detector need to be optimized only for this value. Moreover, at $30 \mathrm{keV}$, muonium formation in the trigger foil is negligible and effects of multiple scattering and energy straggling are reduced.

Since the initial energy spread of the source is small, the energy uncertainty of the tertiary beam is determined essentially by the energy straggling $(\sim 500 \mathrm{eV})$ of the muons when they traverse the foil of the trigger detector. This spread can be reduced by introducing a cylindrical electrode after the foil and pulsing it with the signal from the trigger detector. By choosing an appropriate pulse shape, a sizable reduction of the energy spread can be achieved.

\section{Conclusions}

In conclusion it has been shown that the moderation of a surface $\mu^{+}$beam by condensed van der Waals moderators produces highly polarized slow muons. This and the successful test of a trigger detector for a continuous beam machine demonstrate that a very low energy beam of polarized $\mu^{+}$for thin film and surface $\mu \mathrm{SR}$ can be realized at PSI by making use of the moderation technique. A first $\mu$ SR measurement of a thin sample has been performed.

Before slow $\mu^{+}$can be routinely used for various experiments and a tertiary $\mu^{+}$beam is available, further developments are necessary. For instance, to make efficient use of the muons down to very low energies, an acceleration-deceleration section must be inserted between moderator and sample. Parallel to this the search for moderators with high efficiency is still an important task for the development of slow $\mu^{+}$physics.

\section{Acknowledgement}

We thank B. Patterson, $\mathrm{H}$. Keller and A. Schenck for providing the $\mathrm{SiO}_{2}$ sample and for interesting discussions. We also thank H.-R. Ott, H.-Ch. Walter and G. zu Putlitz for support and encouragement. One of us (TP) thanks the Land Baden-Württemberg for a Fellowship and acknowledges financial support by the Human Capital and Mobility Program (European Union) and by the Swiss Federal Office of Education and Science.

\section{References}

[1] A. Schenck, Muon Spin Rotation Spectroscopy (Hilger, Bristol, 1985).

[2] K. Nagamine, At. Phys. 10 (1987) 225. 
[3] D.R. Harshman, J.B. Warren, J.L. Beveridge, K.R. Kendall, R.F. Kiefl, C.J. Oram, A.P. Mills, W.S. Crane, A.S. Rupaal and J.H. Turner, Phys. Rev. Lett. 56(1986) 2850.

[4] H. Daniel, Z. Phys. A 313 (1983) 249.

[5] D. Taqqu, Nucl. Instr. Meth. A 247 (1986) 288.

[6] E. Morenzoni, in: Future of Muon Physics, eds. K. Jungmann, V.W. Hughes and G. zu Putlitz (Springer, Berlin, 1992) [Suppl. to Z. Phys. C 56 (1992) S243].

[7] D.R. Harshman, A.P. Mills, J.L. Beveridge, K.R. Kendall, G.D. Morris, M. Senba, J.B. Warren, A.S. Rupaal and J.H. Turner, Phys. Rev. B 36 (1987) 8850.

[8] M. Meyberg, E. Morenzoni, Th. Wutzke, F. Kottmann, U. Zimmermann, K. Jungmann, B. Matthias and Th. Prokscha, Hyp. Int. 87 (1994) 1075.

[9] Th. Wutzke, PhD Thesis, ETH-Zürich, Switzerland (1995).

[10] Th. Prokscha, PhD Thesis, University of Heidelberg, Germany (1995).

[11] C. Rau and R. Sizmann, Phys. Lett. 43 A (1973) 317.

[12] E. Morenzoni, F. Kottmann, D. Maden, B. Matthias, M. Meyberg, Th. Prokscha, Th. Wutzke and U. Zimmermann, Phys. Rev. Lett. 72(1994) 2793.

[13] M. Senba, J. Phys. B 21 (1988) 3093; B 22 (1989) 2027.

[14] M.L. Klein and J.A. Venables, eds., Rare Gas Solids, Vol. 2 (Academic Press, New York, 1977).

[15] R.F. Kiefl, J.B. Warren, G.M. Marshall, C.J. Oram and C.W. Clawson, J. Chem. Phys. 74 (1981) 308 .

[16] E. Krasnoperov et al., JETP Lett. 59 (1994) 749.

[17] B.F. Kirillov, B.A. Nikolsky, A.V. Pirogov, V.G. Storchak, V.N. Duginov, V.G. Grebennik, S. Kapusta, A.B. Lazarev, S.N. Shilov and V.A. Zhukov, Hyp. Int. 65 (1990) 819.

[18] D. Hasselkamp and A. Scharmann, Phys. Stat. Sol. (a) 79 (1983) K197. 